\title{
Colonic J-pouch versus side-to-end anastomosis for rectal cancer: a systematic review and meta-analysis of randomized controlled trials
}

\author{
Zheng Wang ${ }^{*}$
}

\begin{abstract}
Background: This study aims to compare colonic J-pouch and side-to-end anastomosis for rectal cancer in terms of surgical and bowel functional outcomes and quality of life (QoL).

Methods: A systematic literature search was performed in PubMed, Embase and Cochrane. The last search was performed on March 28, 2021. All randomized controlled trials comparing colonic J-pouch with side-to-end anastomosis for rectal cancer were enrolled. The main outcomes were bowel functional outcomes and QoL. The secondary outcomes were surgical outcomes including operative time, postoperative hospital stay, complications, and mortality.

Results: Nine articles incorporating 7 trials with a total of 696 patients ( 330 by J-pouch and 366 by side-to-end) were enrolled in this meta-analysis. The bowel functional outcomes were comparable between J-pouch and side-to-end groups in terms of stool frequency, urgency, and incomplete defecation at the short term ( $<8$ months), medium term (8-18 months), and long term (> 18 months) follow up evaluations. No difference was observed between groups with regards to QoL (SF-36: physical function, social function, and general health perception). Besides, surgical outcomes were also similar in two groups.

Conclusion: The currently limited evidence suggests that colonic J-pouch and side-to-end anastomosis are comparable in terms of bowel functional outcomes, QoL, and surgical outcomes. Surgeons may choose either of the two techniques for anastomosis. A large sample randomized controlled study comparing colonic J-pouch and side-to-end anastomosis for rectal cancer is warranted.
\end{abstract}

Keywords: Rectal cancer, Colonic J-pouch anastomosis, Side-to-end anastomosis, Meta-analysis

\section{Background}

Total mesorectal excision (TME) is the best available treatment for rectal cancer. With the advancement of surgical techniques, the majority of patients with mid and upper rectal cancer can undergo a sphincter-saving TME procedure. After TME, the most widely used

*Correspondence: wangzheng@wchscu.cn

Department of Science and Technology, West China Hospital, Sichuan

University, Chengdu, China reconstructive technique is straight coloanal anastomosis. However, because the sigmoid colon is usually excised during surgery which decreases the storage volume of stool, there is a common problem seriously influencing the life quality of patients, including increased tool frequency, urgency and incontinence, which is termed as "anterior resection syndrome (ARS)" [1]. About 19-56\% of patients would suffer from ARS [2-6]. Thus, the demand for a technique with 
better functional outcomes made surgeons modify the straight anastomotic technique.

Colonic J-pouch anastomosis was introduced by Lazorthes et al. [7] and Parc et al. [8] in 1986. A 5-8 cmlong colonic segment was considered as the optimum size of the J-pouch (Fig. 1B) [9]. Previous clinical trials and meta-analyses have proved that J-pouch could provide a better quality of life (QoL) and bowel functional outcomes compared with straight anastomosis $[10,11]$. In some patients with the narrow pelvis or bulky mesentery, however, it is unable to perform colonic J-pouch [12]. Thus, another modified anastomotic technique, side-to-end anastomosis, which has been used since 1966, has gained attention [13]. Side-to-end anastomosis usually needs a 3-5 $\mathrm{cm}$-long colonic segment (Fig. 1A). Multiple studies on the literature have shown that compared with straight anastomosis, side-to-end anastomosis has advantages in bowel functional and operative outcomes [14].

Previous trials [15-18] and meta-analyses [11, 19, 20] including a Cochrane review [11] have shown that J-pouch and side-to-end anastomosis had similar functional outcomes. Recent trials, however, reported that colonic J-pouch could provide a better short-term quality of life [21], but it may increase the anastomotic leakage rate [22]. Up to now, there is no clear evidence on which one of the two anastomotic techniques is better. Therefore, we conducted this meta-analysis to compare surgical and bowel functional outcomes and QoL in patients undergoing resection of a mid to upper rectal cancer with reconstruction using either a colonic J-pouch or a side-to-end pouch.

\section{Methods}

This study was performed according to the recommendations of the preferred reporting items for systematic reviews and meta-analyses statement (PRISMA) [23]. In this study, colonic J-pouch was performed with $4-8 \mathrm{~cm}$ long colonic segment, while side-to-end anastomosis was performed with 3-6 cm-long colonic segment.

\section{Study selection}

A systematic literature search was performed in PubMed, Embase and Cochrane. Our search strategy included terms "rectal cancer, rectal cancers, colorectal cancer, colorectal cancers, rectal carcinoma, rectal carcinomas, colorectal carcinoma, or colorectal carcinomas" and "side-to-end, side to end, end to side, or end-to-side" and "J pouch, J-pouch, colonic-J-pouch, or coloanal-J-pouch". The search details of each electronic database are shown in Supplementary table 1. The last search was performed on March 28, 2021. Furthermore, we also performed a manual search of references of articles and reviews to enrolled additional potentially eligible studies. All randomized controlled trials (RCTs) comparing colonic J-pouch with side-to-end anastomosis for rectal cancer were enrolled. Non-randomized trials, such as retrospective studies, reviews, meta-analyses, and comments, were excluded. If studies were reported on the same population of patients, the results were either combined, or the study with more detailed data was used.

\section{Data extraction}

To avoid any mistakes or omissions, two authors reviewed all identified studies and extracted data using a standard paper-based extraction sheet independently.
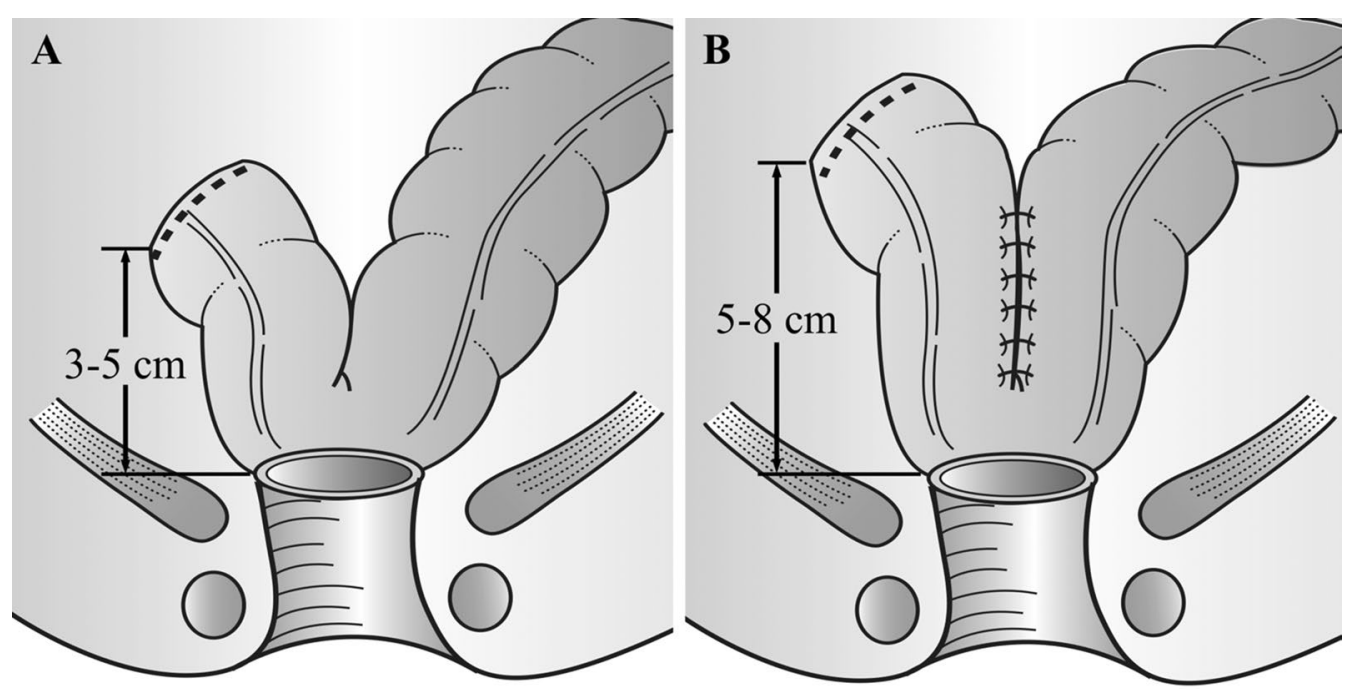

Fig. 1 Side-to-end (A) and colonic J-pouch anastomosis (B) 
Whenever there were disagreements, a third reviewer was needed. The following items were extracted from each study: first author's name, year of publication, the sample size of each arm, gender (male sex), age, the distance of the distal edge of the tumor from the anal verge, the distance of the anastomosis from the anal verge, neoadjuvant/adjuvant therapy, and outcomes of interest.

\section{Outcomes of interest}

The main outcomes were bowel functional outcomes and QoL. Bowel functional outcomes mainly included three indexes: stool frequency, urgency, incomplete defecation and incontinence. According to the previous Cochrane meta-analysis [11], we recorded bowel functional outcomes and QoL at three time periods: short term ( $<8$ months), medium term (8-18 months), and long term ( $>18$ months). The secondary outcomes were surgical outcomes including operative time, postoperative hospital stay, postoperative complications, reoperation, and mortality.

\section{Quality assessment}

We used the modified Jadad score system to assess the methodological quality of the randomized controlled trials (total score, $5 ; 1-2$, low quality; $3-5$, high quality) [24].

\section{Statistical analysis}

We used Review Manager version 5.3 (The Cochrane Collaboration, Software Update, Oxford) for data analyses. $P$ value $<0.05$ was considered statistically significant. Continuous outcomes were analyzed using weighted mean difference (WMD), while dichotomous outcomes were analyzed using odds ratios (OR) or risk ratios (RR). If means and standard deviations (SDs) of continuous outcomes were not provided, we used methods described by Hozo et al. [25] to calculate means and SDs from means and range values or medians and range values. Besides, we used the Chi-squared test and Higgins I-squared test to calculate Heterogeneity. The value of $P<0.05$ and $\mathrm{I}^{2}>50 \%$ was considered as high heterogeneity, and therefore, a random-effects model was applied; otherwise, a fixed-effects model was applied. If high heterogeneity existed, we conducted sensitivity analysis by removing one study each time to decrease heterogeneity. Publication bias was assessed using Begg's funnel plot.

\section{Results}

The flow chart of the literature search is shown in Fig. 2. After duplicate data were removed, there were 71 records. After the initial review, 45 studies were excluded. Besides, one additional article was enrolled through a manual search. Finally, a total of 27 relevant studies were further evaluated. Of those studies, 18 reports were excluded due to the following reasons: four studies did not present sufficient data, eight studies were reviews or meta-analyses, one study was a comment, two studies had overlap patients with similar results, one study reported surgical technique, and two studies were retrospective or prospective study. Of the remaining nine articles, two papers by Machado et al. [16, 18] reported different outcomes which were based on the same trial, as well as another two papers by Marti et al. [26] and Ribi et al. [21]. Thus, nine articles incorporating 7 trials were enrolled in this meta-analysis [15-18, 21, 22, 26-28]. A total of 696 patients ( 330 by colonic J-pouch and 366 by side-to-end) were included.

\section{Patient demographics}

The characteristics of the included studies were summarized in Table 1. No significant differences were found between colonic J-pouch and side-to-end groups in terms of the male sex, the distance of the distal edge of the tumor from the anal verge, the distance of the anastomosis from the anal verge, or neoadjuvant/adjuvant therapy. Although the age of J-pouch patients was younger than that of side-to-end, the mean age difference was only 1.60 $\left[\mathrm{WMD}=-1.60,95 \% \mathrm{CI}(-2.86,0.35), \mathrm{I}^{2}=0 \%, P=0.01\right]$.

\section{Quality of included studies}

Although there were nine articles enrolled, only seven trials were involved. Thus, we just assessed those seven trials through seven articles. Of those seven trials, six trials had high quality, and one trial had low quality according to the modified Jadad score system (Table 2).

\section{Bowel functional outcomes}

The bowel functional outcomes were shown in Table 3.

\section{Baseline}

Three trials comparing J-pouch with side-to-end reported baseline stool frequency [17, 22, 28] and urgency $[17,18,22]$. The pooled data showed that the baseline stool frequency (J-pouch 1.1, side-to-end 1.3) had a random effects model of WMD $=-0.19,95 \% \mathrm{CI}$ $(-0.42,0.03), \mathrm{I}^{2}=95 \%, \mathrm{P}=0.09$ and urgency defined as yes or no (J-pouch $11 \%$, side-to-end $8 \%$ ) had a fixed effects model of OR $=1.32,95 \%$ CI $(0.52,3.39), \mathrm{I}^{2}=19 \%$, $\mathrm{P}=0.56$ which were comparable between the two groups. Only two trials reported baseline incomplete defecation defined as yes or no $[17,18]$, which was also similar in two groups [J-pouch $4 \%$, side-to-end $1 \%$, fixed effects model, OR $\left.=2.39,95 \% \mathrm{CI}(0.34,16.67), \mathrm{I}^{2}=0 \%, P=0.38\right]$. 


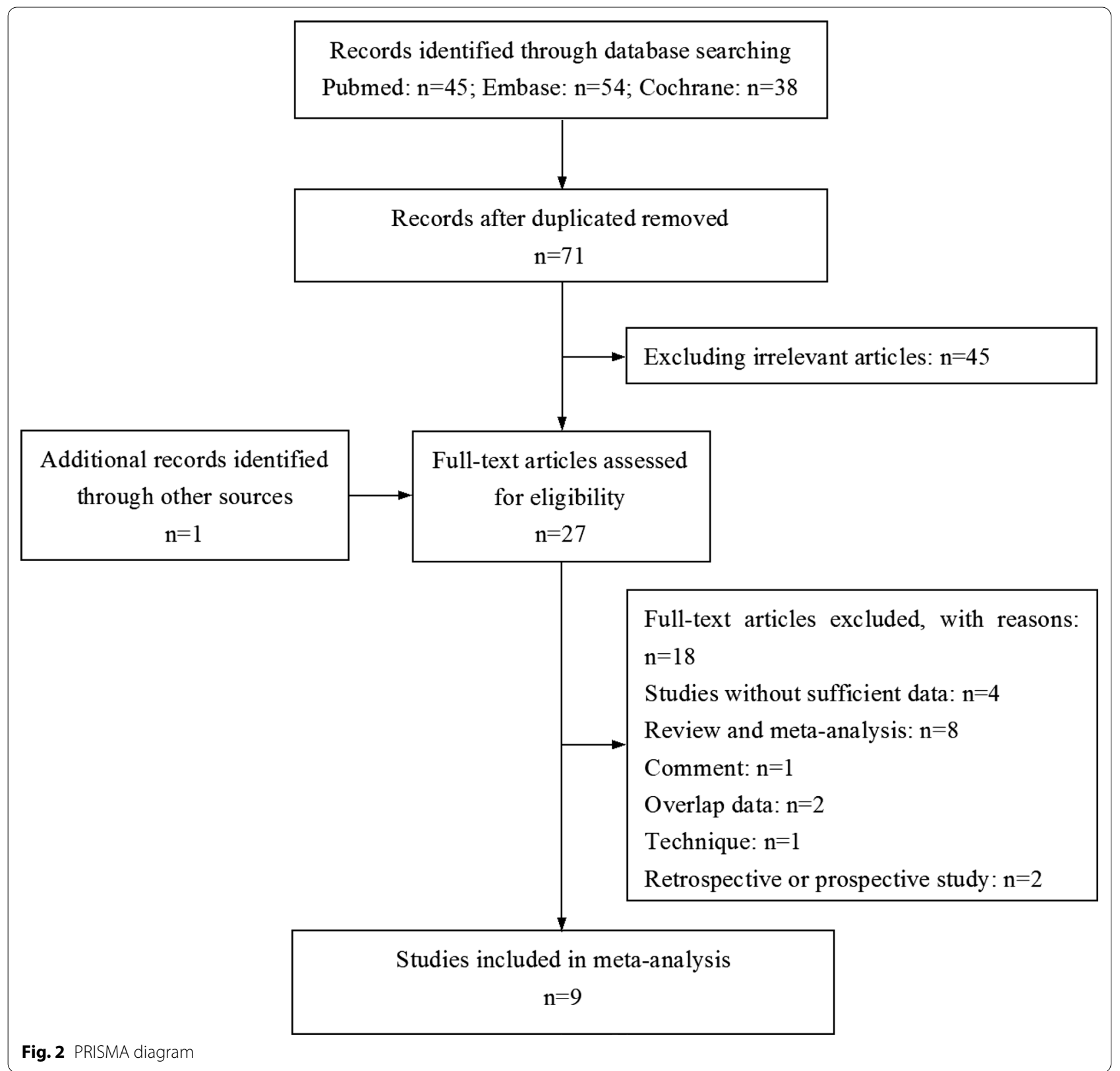

\section{Short term}

Five trials reported stool frequency [17, 18, 22, 27, 28 ], which was comparable between the two groups [J-pouch 2.7, side-to-end 3.0, random effects model, $\left.\mathrm{WMD}=-0.30,95 \% \mathrm{CI}(-1.04,0.44), \mathrm{I}^{2}=95 \%, P=0.42\right]$. The sensitivity analysis could not reduce the high heterogeneity. Four trials reported urgency (yes or no) [17, $18,22,27]$. No significant difference was found between two groups [J-pouch $30 \%$, side-to-end $41 \%$, fixed effects model, OR $=0.53$, 95\% CI $\left.(0.28,1.00), \mathrm{I}^{2}=0 \%, P=0.05\right]$. Three trials reported incomplete defecation (yes or no) $[17,18,27]$, which was also comparable in two groups
[J-pouch 35\%, side-to-end 27\%, fixed effects model, $\left.\mathrm{OR}=1.65,95 \% \mathrm{CI}(0.83,3.28), \mathrm{I}^{2}=0 \%, P=0.15\right]$.

\section{Medium term}

Four trials reported stool frequency [17, 18, 22, 28], which was comparable between the two groups [J-pouch 2.6, side-to-end 2.9, random effects model, WMD $=-0.32$, $95 \%$ CI $\left.(-1.10,0.45), \mathrm{I}^{2}=96 \%, P=0.41\right]$. The sensitivity analysis could not reduce the high heterogeneity. Three trials reported urgency (yes or no) $[17,18,22]$. The pooled data showed no significant difference between two groups [J-pouch 37\%, side-to-end 48\%, fixed effects 
Table 1 Study characteristics

\begin{tabular}{|c|c|c|c|c|c|c|c|c|c|c|c|}
\hline \multirow[t]{2}{*}{ Authors } & \multirow[t]{2}{*}{ No. of center } & \multicolumn{3}{|c|}{ No. of patients } & & \multicolumn{2}{|c|}{ Male } & \multicolumn{2}{|l|}{ Age } & \multicolumn{2}{|c|}{ Tumor from AV } \\
\hline & & $J P$ & & SE & & JP & SE & $\mathrm{JP}$ & SE & $\overline{J P}$ & SE \\
\hline Doeksen & 7 & 55 & & 52 & & 36 & 37 & $61.8 / 12.3$ & $63.8 / 8.8$ & NR & NR \\
\hline Huber & 1 & 29 & & 30 & & 13 & 12 & 62.3 & 61.9 & $5.2 / 1.6$ & $5.8 / 1.5$ \\
\hline Jiang & 1 & 24 & & 24 & & 12 & 15 & $62.3 / 3.3$ & $64.9 / 2.8$ & $7.9 / 1.5$ & $8.6 / 0.3$ \\
\hline Machado & 1 & 50 & & 50 & & 27 & 32 & 63.8/11.3 & $65.0 / 11.8$ & $9.5 / 3.0$ & $9.8 / 2.8$ \\
\hline Marti & 15 & 63 & & 95 & & 38 & 62 & $63.4 / 13.7$ & 63.9/9.5 & $6.0 / 2.5$ & $7.5 / 2.3$ \\
\hline Okkabaz & 1 & 29 & & 28 & & 18 & 19 & $58.9 / 13.7$ & $59.1 / 11.9$ & $7.9 / 3.8$ & $6.2 / 3.8$ \\
\hline Parc & 7 & 80 & & 87 & & 59 & 52 & $60.2 / 9.7$ & $59.6 / 10.6$ & $2.5 / 0.7$ & $2.0 / 0.7$ \\
\hline \multirow[t]{2}{*}{ Authors } & \multicolumn{3}{|c|}{ Anastomosis from AV } & & \multicolumn{3}{|c|}{ Neoadjuvant therapy } & \multicolumn{2}{|c|}{ Adjuvant therapy } & \multirow{2}{*}{\multicolumn{2}{|c|}{$\begin{array}{l}\text { Quality of life } \\
\text { assessment scale }\end{array}$}} \\
\hline & $\mathrm{JP}$ & & SE & & $\mathrm{JP}$ & & SE & $\mathrm{JP}$ & SE & & \\
\hline Doeksen & $N R$ & & NR & & 55 & & 52 & 0 & 0 & & $\begin{array}{l}\text { ORTC- } \\
38\end{array}$ \\
\hline Huber & $3.8 / 0.8$ & & $4.2 / 0.5$ & & NR & & $N R$ & NR & NR & NA & \\
\hline Jiang & $4.8 / 0.2$ & & $5.3 / 0.3$ & & 12 & & 10 & 2 & 1 & NA & \\
\hline Machado & $4.0 / 1.0$ & & $3.8 / 0.8$ & & 39 & & 39 & 4 & 2 & NA & \\
\hline Marti & $N R$ & & NR & & 49 & & 72 & 34 & 53 & FAC & \\
\hline Okkabaz & $N R$ & & NR & & 17 & & 19 & NR & NR & $\mathrm{SF}_{-}-\mathrm{S}$ & $\begin{array}{l}\text { M, FISI, } \\
\text { /F }\end{array}$ \\
\hline Parc & $N R$ & & NR & & 42 & & 50 & NR & NR & & T-C, IIEF, \\
\hline
\end{tabular}

JP J-pouch, SE side-to-end, AV anal verge, NR not report, NA not applicable, COREFO COloREctal Functional Outcome, FACT-C Functional Assessment of Cancer Therapy-Colorectal, SHIM Sexual Health Inventory for Men, FISI Fecal Incontinence Severity Index, FSFI Female Sexual Function Index, OBVF Overactive BladderValidated Form, IIEF International Index of Erectile Function

Table 2 Evaluation of methodological qualities of included randomized controlled trials

\begin{tabular}{|c|c|c|c|c|c|c|c|}
\hline Items/author & Huber & Jiang & Okkabaz & Parc & Doeksen & Machado & Marti \\
\hline Described as randomized & 1 & 1 & 1 & 1 & 1 & 1 & 1 \\
\hline Appropriate randomization method described & 1 & 1 & 1 & 1 & 1 & 1 & 1 \\
\hline Subject blinded to intervention & 0 & 0 & 1 & 0 & 0 & 0 & 0 \\
\hline Evaluator blinded to intervention & 0 & 0 & 0 & 0 & 0 & 0 & 0 \\
\hline Description of withdrawals and dropouts & 0 & 1 & 1 & 1 & 1 & 1 & 1 \\
\hline Score & 2 & 3 & 4 & 3 & 3 & 3 & 3 \\
\hline
\end{tabular}

Methodological qualities of included randomized controlled trials were assessed by modified Jadad score system. Total score, $5 ; 1-2$, low quality trial; $3-5$, high quality trial

model, $\left.\mathrm{OR}=0.67,95 \% \mathrm{CI}(0.34,1.29), \mathrm{I}^{2}=42 \%, P=0.23\right]$. Two trials reported incomplete defecation (yes or no) [17, 18 ], which was also comparable in two groups [J-pouch $36 \%$, side-to-end $51 \%$, fixed effects model, $\mathrm{OR}=0.56$, $95 \%$ CI $\left.(0.28,1.12), \mathrm{I}^{2}=31 \%, P=0.10\right]$.

\section{Long term}

Three trials reported stool frequency [16, 17, 28], which was comparable between the two groups [J-pouch 1.4, side-to-end 1.8 , random effects model, WMD $=-0.34$,
95\% CI $\left.(-0.84,0.16), \mathrm{I}^{2}=91 \%, P=0.19\right]$. The sensitivity analysis could not reduce the high heterogeneity. Two trials reported urgency (yes or no) [16, 17]. The pooled data showed no significant difference between two groups [J-pouch 22\%, side-to-end 39\%, fixed effects model, $\left.\mathrm{OR}=0.40,95 \% \mathrm{CI}(0.16,1.01), \mathrm{I}^{2}=0 \%, P=0.05\right]$. Two trials reported incomplete defecation (yes or no) $[16,17]$, which was also comparable in two groups [J-pouch $33 \%$, side-to-end $39 \%$, fixed effects model, $\mathrm{OR}=0.71,95 \% \mathrm{CI}$ $\left.(0.31,1.62), \mathrm{I}^{2}=0 \%, P=0.41\right]$. 
Table 3 Bowel functional outcomes

\begin{tabular}{|c|c|c|c|c|c|c|c|c|c|c|}
\hline \multirow{2}{*}{$\begin{array}{l}\text { Bowel functional } \\
\text { outcomes }\end{array}$} & \multirow{2}{*}{$\begin{array}{l}\text { No. of } \\
\text { studies }\end{array}$} & \multicolumn{2}{|l|}{ J-pouch } & \multicolumn{2}{|c|}{ Side-to-end } & \multirow[t]{2}{*}{$I^{2}(\%)$} & \multirow[t]{2}{*}{ Analysis model } & \multirow[t]{2}{*}{ OR/WMD } & \multirow[t]{2}{*}{$95 \% \mathrm{Cl}$} & \multirow[t]{2}{*}{$P$ value } \\
\hline & & Patients & Mean/rate & Patients & Mean/rate & & & & & \\
\hline \multicolumn{11}{|l|}{ Baseline } \\
\hline Stool frequency & 3 & 122 & $1.1^{\#}$ & 134 & $1.3^{\#}$ & 71 & Random & $-0.19^{\dagger}$ & $-0.42,0.03$ & 0.09 \\
\hline Urgency & 3 & 92 & $11 \%^{\wedge}$ & 97 & $8 \%^{\wedge}$ & 19 & Fixed & $1.32^{*}$ & $0.52,3.39$ & 0.56 \\
\hline Incomplete defecation & 2 & 74 & $4 \%^{\wedge}$ & 74 & $1 \% \wedge$ & 0 & Fixed & $2.39^{*}$ & $0.34,16.67$ & 0.38 \\
\hline \multicolumn{11}{|l|}{ Short term } \\
\hline Stool frequency & 5 & 195 & $2.7^{\#}$ & 208 & $3.0^{\#}$ & 95 & Random & $-0.30^{\dagger}$ & $-1.04,0.44$ & 0.42 \\
\hline Urgency & 4 & 115 & $30 \%^{\wedge}$ & 121 & $41 \%^{\wedge}$ & 0 & Fixed & $0.53^{*}$ & $0.28,1.00$ & 0.05 \\
\hline Incomplete defecation & 3 & 97 & $35 \%^{\wedge}$ & 98 & $27 \%^{\wedge}$ & 0 & Fixed & $1.65^{*}$ & $0.83,3.28$ & 0.15 \\
\hline \multicolumn{11}{|l|}{ Medium term } \\
\hline Stool frequency & 4 & 164 & $2.6^{\#}$ & 173 & $2.9^{\#}$ & 96 & Random & $-0.32^{\dagger}$ & $-1.10,0.45$ & 0.41 \\
\hline Urgency & 3 & 84 & $37 \%^{\wedge}$ & 86 & $48 \%^{\wedge}$ & 42 & Fixed & $0.67^{*}$ & $0.34,1.29$ & 0.23 \\
\hline Incomplete defecation & 2 & 66 & $36 \%^{\wedge}$ & 63 & $51 \%^{\wedge}$ & 31 & Fixed & $0.56^{*}$ & $0.28,1.12$ & 0.10 \\
\hline \multicolumn{11}{|l|}{ Long term } \\
\hline Stool frequency & 3 & 135 & $1.4^{\#}$ & 138 & $1.8^{\#}$ & 91 & Random & $-0.34^{\dagger}$ & $-0.84,0.16$ & 0.19 \\
\hline Urgency & 2 & 55 & $22 \%^{\wedge}$ & 51 & $39 \%^{\wedge}$ & 0 & Fixed & $0.40^{*}$ & $0.16,1.01$ & 0.05 \\
\hline Incomplete defecation & 2 & 55 & $33 \%^{\wedge}$ & 51 & $39 \% \wedge$ & 0 & Fixed & $0.71^{*}$ & $0.31,1.62$ & 0.41 \\
\hline
\end{tabular}

$\mathrm{OR}$ odds ratio, " pooled mean, ^ pooled rate, WMD weighted mean difference, $\mathrm{Cl}$ confidence interval, ${ }^{*} \mathrm{OR},{ }^{\dagger} \mathrm{WMD}$

Table 4 Quality of life (SF-36)

\begin{tabular}{|c|c|c|c|c|c|c|c|c|c|c|}
\hline \multirow[t]{2}{*}{ Quality of life (SF-36) } & \multirow[t]{2}{*}{ No. of studies } & \multicolumn{2}{|l|}{ J-pouch } & \multicolumn{2}{|c|}{ Side-to-end } & \multirow[t]{2}{*}{$I^{2}(\%)$} & \multirow[t]{2}{*}{ Analysis model } & \multirow[t]{2}{*}{ WMD } & \multirow[t]{2}{*}{$95 \% \mathrm{Cl}$} & \multirow[t]{2}{*}{ P value } \\
\hline & & Patients & Mean & Patients & Mean & & & & & \\
\hline \multicolumn{11}{|l|}{ Baseline } \\
\hline Physical function & 2 & 63 & 81.09 & 64 & 75.75 & 0 & Fixed & 5.34 & $-1.61,12.28$ & 0.13 \\
\hline Social function & 2 & 66 & 74.76 & 66 & 70.93 & 0 & Fixed & 3.83 & $-4.56,12.21$ & 0.37 \\
\hline General health perception & 2 & 65 & 67.63 & 65 & 60.19 & 0 & Fixed & 7.44 & $0.39,14.48$ & 0.04 \\
\hline \multicolumn{11}{|l|}{ Short term } \\
\hline Physical function & 2 & 39 & 75.85 & 46 & 69.28 & 82 & Random & 6.57 & $-14.96,28.10$ & 0.55 \\
\hline Social function & 2 & 40 & 68.91 & 46 & 63.71 & 67 & Random & 5.20 & $-12.47,22.88$ & 0.56 \\
\hline General health perception & 2 & 39 & 70.08 & 47 & 70.56 & 79 & Random & -0.48 & $-19.39,18.43$ & 0.96 \\
\hline \multicolumn{11}{|l|}{ Medium term } \\
\hline Physical function & 2 & 46 & 84.13 & 44 & 77.58 & 66 & Random & 6.55 & $-10.59,23.69$ & 0.45 \\
\hline Social function & 2 & 48 & 82.94 & 45 & 82.76 & 70 & Random & 0.18 & $-14.64,15.00$ & 0.98 \\
\hline General health perception & 2 & 48 & 71.75 & 45 & 70.81 & 72 & Random & 0.94 & $-12.62,14.51$ & 0.89 \\
\hline
\end{tabular}

WMD weighted mean difference, $\mathrm{Cl}$ confidence interval

\section{Quality of life}

Different trials used different questionnaires to assess QoL (Table 1). Only two trials [15, 22] which both used SF-36 questionnaire could be pooled together and these two trials involving 164 patients ( 84 by colonic J-pouch and 80 by side-to-end) in total served as our evaluation of QoL in this study. The SF-36 outcomes were shown in Table 4. At the baseline, the physical function and social function were comparable between J-pouch and side-to-end groups; however, the general health perception was slightly better in J-pouch [fixed effects model, $\left.\mathrm{WMD}=7.44,95 \% \mathrm{CI}(0.39,14.48), \mathrm{I}^{2}=0 \%, P=0.04\right]$. At short term, the physical function [random effects model, $\left.\mathrm{WMD}=6.57,95 \% \mathrm{CI}(-14.96,28.10), \mathrm{I}^{2}=82 \%, P=0.55\right]$, social function [random effects model, WMD $=5.20$, $95 \%$ CI $\left.(-12.47,22.88), \mathrm{I}^{2}=67 \%, P=0.56\right]$, and general 


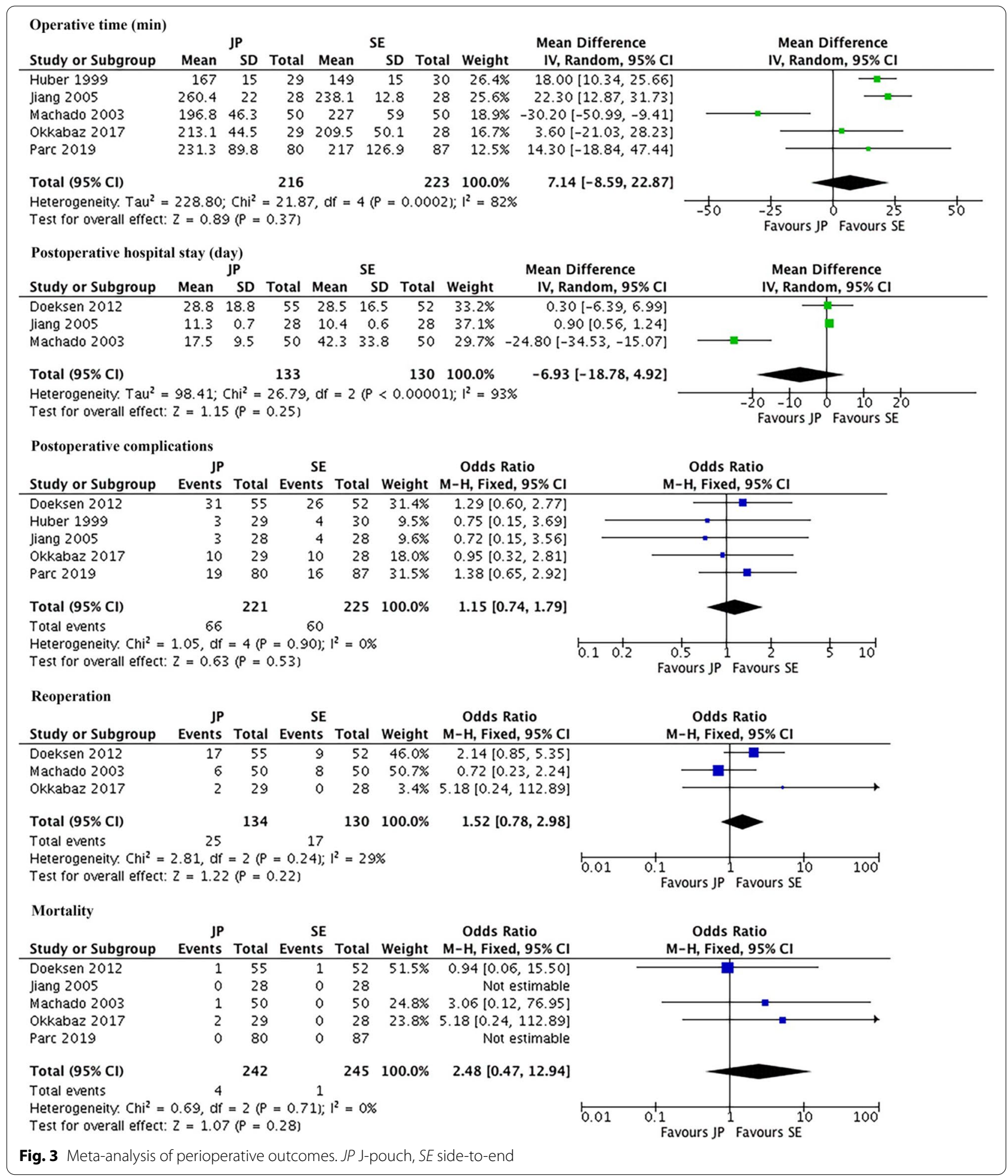

health perception [random effects model, WMD $=-0.48$, $95 \%$ CI $\left.(-19.39,18.43), I^{2}=79 \%, P=0.96\right]$ were all similar in two groups. At medium term, there were no significant difference between J-pouch and side-to-end groups in terms of physical function [random effects model, $\left.\mathrm{WMD}=6.55,95 \% \mathrm{CI}(-10.59,23.69), \mathrm{I}^{2}=66 \%, P=0.45\right]$, social function [random effects model, WMD $=0.18$, $95 \%$ CI $\left.(-14.64,15.00), \mathrm{I}^{2}=70 \%, P=0.98\right]$, and general 


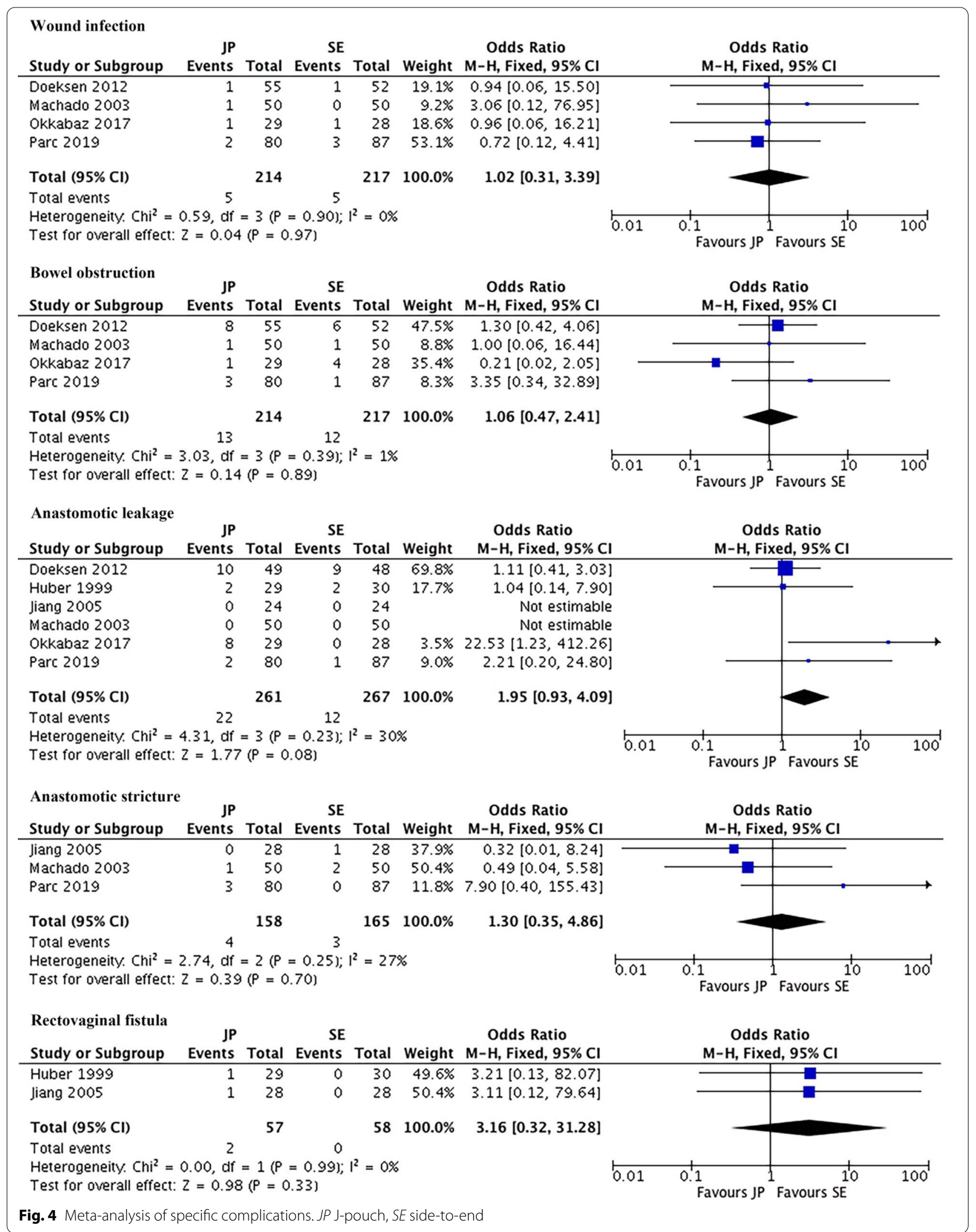


health perception [random effects model, WMD $=0.94$, $95 \%$ CI $\left.(-12.62,14.51), I^{2}=72 \%, P=0.89\right]$. The sensitivity analysis could not reduce the high heterogeneity.

\section{Surgical outcomes}

Figures 3, 4 showed surgical outcomes. The pooled mean operative time was 216 min in J-pouch group and 209 min in side-to-end group. The pooled mean postoperative hospital stay was 20.9 days in J-pouch group and 27.8 days in side-to-end group. There was no statistically significance between the two groups in terms of operative time [random effects model, WMD $=7.14,95 \% \mathrm{CI}$ ( $\left.-8.59,22.87), \mathrm{I}^{2}=82 \%, P=0.37\right]$ and postoperative hospital stay [random effects model, WMD $=-6.93$, $95 \%$ CI $\left.(-18.78,4.92), \mathrm{I}^{2}=93 \%, P=0.25\right]$. The sensitivity analysis could not reduce the high heterogeneity. The pooled postoperative complication rate, reoperation rate, and mortality rate were $29.9 \%, 18.7 \%$, and $1.7 \%$ in J-pouch group and $26.7 \%, 13.1 \%$, and $0.4 \%$ in sideto-end group, respectively. The postoperative complications [fixed effects model, OR $=1.15,95 \%$ CI $(0.74$, $1.79), \mathrm{I}^{2}=0 \%, P=0.53$ ], reoperation [fixed effects model, $\left.\mathrm{OR}=1.52,95 \% \mathrm{CI}(0.78,2.98), \mathrm{I}^{2}=29 \%, P=0.22\right]$ and mortality [fixed effects model, $\mathrm{RR}=2.48,95 \%$ CI $(0.48$, 12.21), $\mathrm{I}^{2}=0 \%, P=0.28$ ] were all comparable between two groups (Fig. 3). As for specific complications, pooled data showed that wound infection [fixed effects model, $\mathrm{OR}=1.02$, 95\% CI $\left.(0.31,3.39), \mathrm{I}^{2}=0 \%, P=0.97\right]$, bowel obstruction [fixed effects model, OR $=1.06,95 \% \mathrm{CI}$ $(0.47,2.41), \mathrm{I}^{2}=1 \%, P=0.89$ ], anastomotic leakage [fixed effects model, OR $=1.95,95 \%$ CI $(0.93,4.09), \mathrm{I}^{2}=30 \%$, $P=0.08$ ], anastomotic stricture [fixed effects model, $\mathrm{OR}=1.30,95 \%$ CI $\left.(0.35,4.86), \mathrm{I}^{2}=27 \%, P=0.70\right]$, and rectovaginal fistula [fixed effects model, OR $=3.16,95 \%$ CI $\left.(0.32,31.28), \mathrm{I}^{2}=0 \%, P=0.33\right]$ were similar in two groups (Fig. 4).

\section{Publication bias}

Publication bias was assessed based on postoperative complications (Fig. 5). There was no obvious publication bias among the studies according to the Begg's funnel plot.

\section{Discussion}

With the advancement of surgical technique, the local recurrence rate after rectal cancer surgery has been decreased from $25-50 \%$ to $3-8 \%$ [29-32]. Naturally, it is time to focus on how to improve bowel functional outcomes and QoL for rectal cancer patients. One of the approaches is to modify the reconstructive technique. Though the most widely used reconstructive technique is the straight coloanal anastomosis, two modified reconstructive techniques, colonic J-pouch and side-to-end, can do provide better QoL and bowel functional outcomes compared with straight anastomosis $[10,11,14]$. Although the length of the colonic segment used for

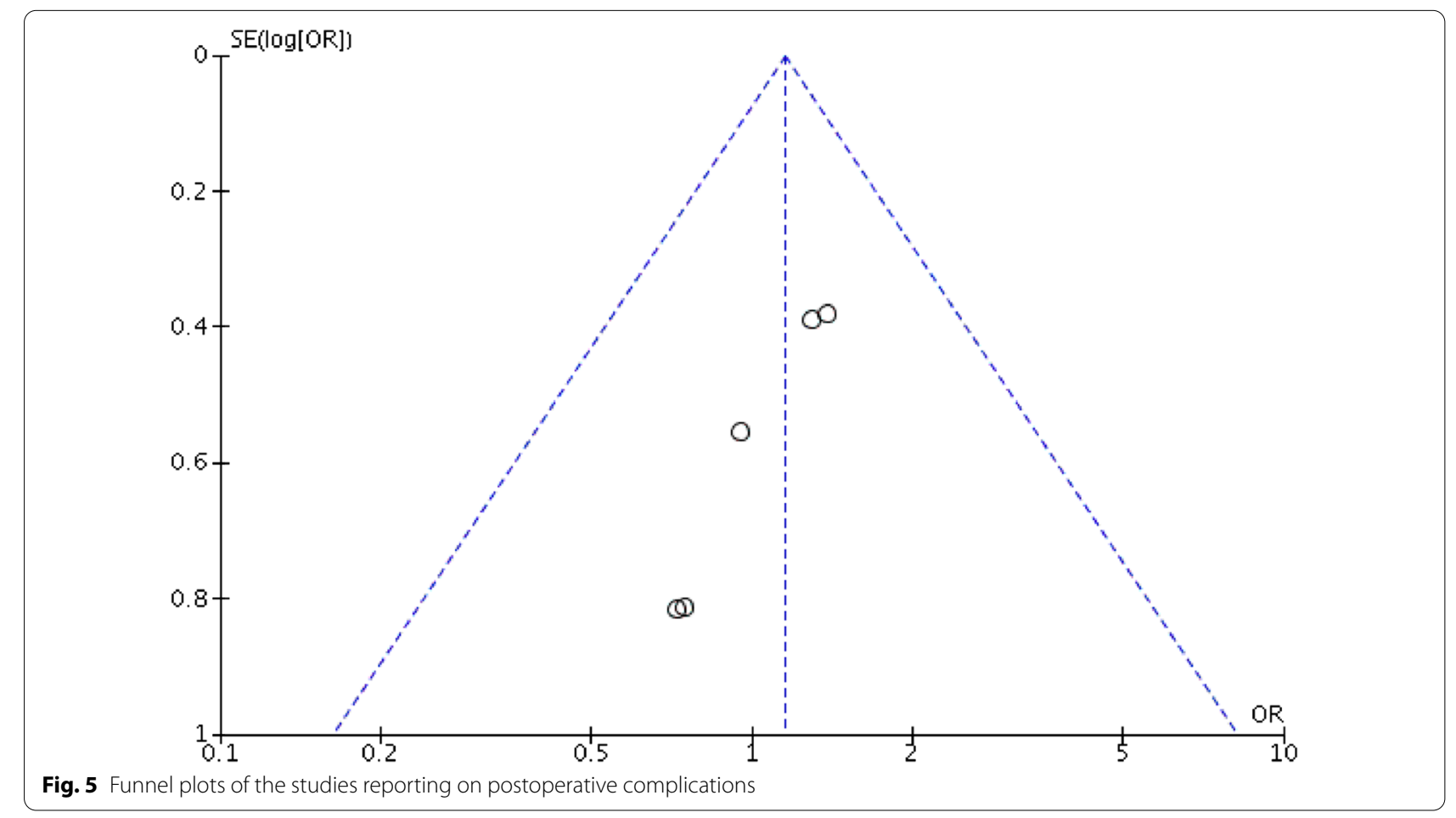


anastomosis was different in varied regions, the colonic J-pouch anastomosis usually needs a 5-8 cm-long colonic segment to build the J-pouch, while the side-toend anastomosis needs a 3-5 cm-long colonic segment (Fig. 1). At present, there is no clear evidence on which one of the two modified anastomotic techniques is better. This meta-analysis enrolling nine articles incorporating 7 trials with a total of 696 patients (330 by J-pouch and 366 by side-to-end) showed that J-pouch was comparable with side-to-end anastomosis in terms of bowel functional outcomes, QoL (SF-36), and surgical outcomes.

Bowel dysfunction is one of the main concerns after sphincter-saving TME surgery. Huber et al. have reported that stool frequency was lower at 6 month after surgery in the J-pouch group [27], however, another trial by Jiang et al. reported it was higher at 6 month in the J-pouch group [17]. Our meta-analysis using only RCTs showed that there was no difference between two groups in terms of stool frequency at all three time-periods, as well as urgency, or incomplete defecation, which were supported by previous meta-analysis [19, 20, 33]. Six studies reported the incontinence outcome, however, the measurement method and/or the data type of incontinence was different $[15-18,26,28]$, which we could not pool together. The meta-analysis by Siddiqui et al. have analyzed the pressure and volumetric outcomes and documented that those results were comparable in J-pouch and side-to-end groups [20]. After that, no recent trial reported pressure or volumetric outcomes. Therefore, we did not analyze those two outcomes.

In relation to QoL, different trials used different questionnaires, including COloREctal Functional Outcome (COREFO) [15], EORTC-QLQ-CR38 [15], SF-36 [15, 22], Functional Assessment of Cancer Therapy-Colorectal (FACT-C) [26, 28], Sexual Health Inventory for Men (SHIM) [22], Fecal Incontinence Severity Index (FISI) [22, 28], Female Sexual Function Index (FSFI) [22], Overactive Bladder-Validated Form (OBVF) [22], SF-12 [28], and International Index of Erectile Function (IIEF) [28]. According to data provided by those trials, we could only analyze the SF-36 outcomes (physical function, social function and general health perception), and the pooled data showed no significant difference was observed at short or medium term between two groups. Previous literatures documented that bowel dysfunction can influence QoL after surgery for rectal cancer [34-36]. As this meta-analysis showed no significant difference existed between J-pouch and side-toend in terms of bowel functional outcomes, it was not surprising that physical function, social function and general health perception were comparable in two groups.

Theoretically, J-pouch anastomosis is technically more demanding, the formation of which should take more time. However, the operative time was comparable in J-pouch and side-to-end groups, which was also supported by previous meta-analysis [20]. The possible reason is that the J-pouch is performed using a stapler, which only takes very little time. No significant difference was observed in the postoperative hospital stay. The postoperative complication rates of enrolled studies were $10.3-56.4 \%$ in the J-pouch group and $13.3-50.0 \%$ in the side-to-end group. The big difference in complication rates was due to the different diagnostic criteria in different departments. The pooled postoperative complication rate was $29.9 \%$ and $26.7 \%$ in J-pouch and side-to-end groups, respectively, which was similar in the two groups. As for anastomotic leakage rate, previous literatures have reported that the incidence of anastomotic leakage ranges from 1 to $21 \%$ in patients undergoing a coloanal anastomosis [37-41]. In our meta-analysis, the anastomotic leakage rate was comparable in two groups $(8.4 \%$ in J-pouch and $4.5 \%$ in side-to-end, $\mathrm{P}=0.08$ ), which was also supported by previous meta-analysis [19]. Other complications (wound infection, bowel obstruction, anastomotic stricture, and rectovaginal fistula) were comparable between J-pouch and side-to-end groups.

Two meta-analyses have shown that J-pouch and sideto-end anastomosis had similar functional outcomes [19, 33]; after that, another three RCTs comparing colonic J-pouch and side-to-end have published recently with controversial results [21, 22, 26, 28]. Therefore, we conducted this update meta-analysis. Although the current analysis still included a relatively small amount of RCTs as well as a small number of patients, it provided valuable data for comparing the colonic J-pouch and side-to-end anastomosis after sphincter-saving TME surgery, considering that there was no large cohort study (more than 100 patients in each group). However, this meta-analysis has some limitations need to be highlighted. First of all, high heterogeneity existed in some analyses, and the sensitivity analysis could not reduce those heterogeneities. The heterogeneity might be influenced by some factors, such as the patient's sex, the experience of the surgeon, the surgical technique (open/laparoscopic), and (neo) adjuvant therapy. Second, there were some completed trials in clinical trial registration, however, the results of those trials were not published, which could induce some bias. Third, different trials used different QoL questionnaires due to the lack of good QoL parameters, we could only analyze the SF-36 outcomes in 2 studies. Fourth, none of these nine studies reported the outcomes separately for men and women; thus, we could not analyze this outcome. Fifth, we did not analyze the nighttime incontinence because no studies reported this outcome. We hope that future studies would address these issues. 


\section{Conclusions}

The currently limited evidence suggests that colonic $\mathrm{J}$-pouch and side-to-end anastomosis are comparable in terms of bowel functional outcomes, QoL, and perioperative outcomes. Hence, surgeons may choose either of the two techniques for anastomosis. A large sample randomized controlled study comparing colonic J-pouch and side-to-end anastomosis for rectal cancer is warranted.

\section{Abbreviations}

ARS: Anterior resection syndrome; OR: Odds ratios; PRISMA: Preferred reporting items for systematic reviews and meta-analyses statement; QoL: Quality of life; RR: Risk ratios; SD: Standard deviation; TME: Total mesorectal excision; WMD: Weighted mean difference; RCTs: Randomized controlled trials.

\section{Acknowledgements}

None.

\section{Authors' contributions}

ZW collected the literatures, analyzed the data and wrote the manuscript. The author read and approved the final manuscript.

\section{Funding}

Not funding was received for this study.

\section{Availability of data and materials}

The dataset used and/or analyzed during the current study are available from the corresponding author on reasonable request.

\section{Declarations}

Ethics approval and consent to participate

Not applicable.

\section{Consent for publication}

Not applicable.

\section{Competing interests}

The author declares no competing interests.

Received: 20 December 2019 Accepted: 29 June 2021

Published online: 21 August 2021

\section{References}

1. Ortiz H, Armendariz P. Anterior resection: do the patients perceive any clinical benefit? Int J Colorectal Dis. 1996;11(4):191-5.

2. Bregendahl S, Emmertsen KJ, Lous J, Laurberg S. Bowel dysfunction after low anterior resection with and without neoadjuvant therapy for rectal cancer: a population-based cross-sectional study. Colorectal Dis. 2013;15(9):1130-9.

3. Juul T, Ahlberg M, Biondo S, et al. Low anterior resection syndrome and quality of life: an international multicenter study. Dis Colon Rectum. 2014;57(5):585-91.

4. Juul T, Battersby NJ, Christensen P, et al. Validation of the English translation of the low anterior resection syndrome score. Colorectal Dis. 2015;17(10):908-16.

5. Ekkarat P, Boonpipattanapong T, Tantiphlachiva K, Sangkhathat S. Factors determining low anterior resection syndrome after rectal cancer resection: a study in Thai patients. Asian J Surg. 2016;39(4):225-31.

6. Hughes DL, Cornish J, Morris C. Functional outcome following rectal surgery-predisposing factors for low anterior resection syndrome. Int J Colorectal Dis. 2017;32(5):691-7.
7. Lazorthes F, Fages P, Chiotasso P, Lemozy J, Bloom E. Resection of the rectum with construction of a colonic reservoir and colo-anal anastomosis for carcinoma of the rectum. Br J Surg. 1986;73(2):136-8.

8. Parc R, Tiret E, Frileux P, Moszkowski E, Loygue J. Resection and coloanal anastomosis with colonic reservoir for rectal carcinoma. Br J Surg. 1986;73(2):139-41.

9. Hida J, Yasutomi M, Fujimoto K, et al. Functional outcome after low anterior resection with low anastomosis for rectal cancer using the colonic J-pouch. Prospective randomized study for determination of optimum pouch size. Dis Colon Rectum. 1996;39(9):986-91.

10. Otto S, Kroesen AJ, Hotz HG, Buhr HJ, Kruschewski M. Effect of anastomosis level on continence performance and quality of life after colonic J-pouch reconstruction. Dig Dis Sci. 2008;53(1):14-20.

11. Brown CJ, Fenech DS, McLeod RS. Reconstructive techniques after rectal resection for rectal cancer. Cochrane Database Syst Rev. 2008;2:CD006040.

12. Harris GJ, Lavery IJ, Fazio VW. Reasons for failure to construct the colonic J-pouch. What can be done to improve the size of the neorectal reservoir should it occur? Dis Colon Rectum. 2002;45(10):1304-8.

13. Zehnder MA. 2 variations of the sigmoid-rectum resection suture. I. Sideto-end anastomosis of the sigmoid-rectum. II. Extraperitoneal transposition of the colonic resection suture. Helv Chir Acta. 1968;35(5):526-34

14. Zhang YC, Jin XD, Zhang YT, Wang ZQ. Better functional outcome provided by short-armed sigmoid colon-rectal side-to-end anastomosis after laparoscopic low anterior resection: a match-paired retrospective study from China. Int J Colorectal Dis. 2012;27(4):535-41.

15. Doeksen A, Bakx R, Vincent $A$, et al. J-pouch vs side-to-end coloanal anastomosis after preoperative radiotherapy and total mesorectal excision for rectal cancer: a multicentre randomized trial. Colorectal Dis. 2012;14(6):705-13.

16. Machado M, Nygren J, Goldman S, Ljungqvist O. Functional and physiologic assessment of the colonic reservoir or side-to-end anastomosis after low anterior resection for rectal cancer: a two-year follow-up. Dis Colon Rectum. 2005;48(1):29-36.

17. Jiang JK, Yang SH, Lin JK. Transabdominal anastomosis after low anterior resection: a prospective, randomized, controlled trial comparing longterm results between side-to-end anastomosis and colonic J-pouch. Dis Colon Rectum. 2005:48(11):2100-8.

18. Machado M, Nygren J, Goldman S, Ljungqvist O. Similar outcome after colonic pouch and side-to-end anastomosis in low anterior resection for rectal cancer: a prospective randomized trial. Ann Surg. 2003:238(2):214-20.

19. Huttner FJ, Tenckhoff $S$, Jensen $K$, et al. Meta-analysis of reconstruction techniques after low anterior resection for rectal cancer. Br J Surg. 2015;102(7):735-45.

20. Siddiqui MRS, Sajid MS, Woods WGA, Cheek E, Baig MK. A meta-analysis comparing side to end with colonic J-pouch formation after anterior resection for rectal cancer. Tech Coloproctol. 2010;14(2):113-23.

21. Ribi K, Marti WR, Bernhard J, et al. Quality of life after total mesorectal excision and rectal replacement: comparing side-to-end, colon J-pouch and straight colorectal reconstruction in a randomized, phase III trial (SAKK 40/04). Ann Surg Oncol. 2019;26(11):3568-76.

22. Okkabaz N, Haksal M, Atici AE, et al. J-pouch vs. side-to-end anastomosis after hand-assisted laparoscopic low anterior resection for rectal cancer: a prospective randomized trial on short and long term outcomes including life quality and functional results. Int J Surg. 2017;47:4-12.

23. Liberati A, Altman DG, Tetzlaff J, et al. The PRISMA statement for reporting systematic reviews and meta-analyses of studies that evaluate healthcare interventions: explanation and elaboration. BMJ. 2009;339:2700.

24. Lam TH, Chung KF, Yeung WF, Yu BY, Yung KP, Ng TH. Hypnotherapy for insomnia: a systematic review and meta-analysis of randomized controlled trials. Complement Ther Me. 2015;23(5):719-32.

25. Hozo SP, Djulbegovic B, Hozo I. Estimating the mean and variance from the median, range, and the size of a sample. BMC Med Res Methodol. 2005;5:13.

26. Marti WR, Curti G, Wehrli H, et al. Clinical outcome after rectal replacement with side-to-end, colon-J-pouch, or straight colorectal anastomosis following total mesorectal excision: a Swiss prospective, randomized, multicenter trial (SAKK 40/04). Ann Surg. 2019;269(5):827-35.

27. Huber FT, Herter B, Siewert JR. Colonic pouch vs side-to-end anastomosis in low anterior resection. Dis Colon Rectum. 1999;42(7):896-902. 
28. Parc Y, Ruppert R, Fuerst A, et al. Better function with a colonic J-pouch or a side-to-end anastomosis?: A randomized controlled trial to compare the complications, functional outcome, and quality of Life in patients with low rectal cancer after a J-pouch or a side-to-end anastomosis. Ann Surg. 2019;269(5):815-26.

29. MacFarlane JK, Ryall RD, Heald RJ. Mesorectal excision for rectal cancer. Lancet. 1993;341(8843):457-60.

30. McCall $\mathrm{L}$, , Cox MR, Wattchow DA. Analysis of local recurrence rates after surgery alone for rectal cancer. Int J Colorectal Dis. 1995;10(3):126-32.

31. Heald RJ, Moran BJ, Ryall RD, Sexton R, MacFarlane JK. Rectal cancer: the Basingstoke experience of total mesorectal excision, 1978-1997. Arch Surg. 1998;133(8):894-9.

32. Carlsen E, Schlichting E, Guldvog I, Johnson E, Heald RJ. Effect of the introduction of total mesorectal excision for the treatment of rectal cancer. Br J Surg. 1998;85(4):526-9.

33. Si C, Zhang Y, Sun P. Colonic J-pouch versus Baker type for rectal reconstruction after anterior resection of rectal cancer. Scand J Gastroenterol. 2013;48(12):1428-35.

34. Pucciarelli S, Del Bianco P, Toppan P, et al. Health-related quality of life outcomes in disease-free survivors of mid-low rectal cancer after curative surgery. Ann Surg Oncol. 2008;15(7):1846-54.

35. Sideris L, Zenasni F, Vernerey D, et al. Quality of life of patients operated on for low rectal cancer: impact of the type of surgery and patients characteristics. Dis Colon Rectum. 2005;48(12):2180-91.
36. Maris A, Penninckx F, Devreese AM, et al. Persisting anorectal dysfunction after rectal cancer surgery. Colorectal Dis. 2013;15(11):12291.

37. Matsubara N, Miyata H, Gotoh M, et al. Mortality after common rectal surgery in Japan: a study on low anterior resection from a newly established nationwide large-scale clinical database. Dis Colon Rectum. 2014;57(9):1075-81.

38. den Dulk M, Marijnen CA, Collette L, et al. Multicentre analysis of oncological and survival outcomes following anastomotic leakage after rectal cancer surgery. Br J Surg. 2009;96(9):1066-75.

39. Branagan G, Finnis D. Prognosis after anastomotic leakage in colorectal surgery. Dis Colon Rectum. 2005;48(5):1021-6.

40. Park JS, Choi GS, Kim SH, et al. Multicenter analysis of risk factors for anastomotic leakage after laparoscopic rectal cancer excision: the Korean laparoscopic colorectal surgery study group. Ann Surg. 2013;257(4):665-71.

41. Maggiori L, Bretagnol F, Lefevre JH, Ferron M, Vicaut E, Panis Y. Conservative management is associated with a decreased risk of definitive stoma after anastomotic leakage complicating sphincter-saving resection for rectal cancer. Colorectal Dis. 2011;13(6):632-7.

\section{Publisher's Note}

Springer Nature remains neutral with regard to jurisdictional claims in published maps and institutional affiliations.
Ready to submit your research? Choose BMC and benefit from:

- fast, convenient online submission

- thorough peer review by experienced researchers in your field

- rapid publication on acceptance

- support for research data, including large and complex data types

- gold Open Access which fosters wider collaboration and increased citations

- maximum visibility for your research: over 100M website views per year

At BMC, research is always in progress.

Learn more biomedcentral.com/submissions 